\title{
Desarrollo de prototipo de estación de maquinado CNC
}

\section{Development of CNC machining station prototype}

JARA-RUIZ, Ricardo†*，RODRÍGUEZ-FRANCO, Martín Eduardo, LÓPEZ-ÁLVAREZ, Yadira Fabiola y LÓPEZ-OLMEDO, Leonardo Joaquín

\author{
Universidad Tecnológica del Norte de Aguascalientes, Av. Universidad No. 1001, Estación Rincón, Rincón de Romos, Ags. \\ C.P. 20400
}

ID $1^{\text {er }}$ Autor: Ricardo, Jara-Ruiz / ORC ID: 0000-0001-7725-4138, Researcher ID Thomson: T-1532-2018, CVU CONACYT ID: 630276

ID $1^{\text {er }}$ Coautor: Martín Eduardo, Rodríguez-Franco / ORC ID: 0000-0002-6804-4777, Researcher ID Thomson: T-15392018, CVU CONACYT ID: 660892

ID $2^{\text {do }}$ Coautor: Yadira Fabiola, López-Álvarez / ORC ID: 0000-0002-9041-1908, Researcher ID Thomson: T-1555-2018, CVU CONACYT ID: 375952

ID $3^{\text {er }}$ Coautor: Leonardo Joaquín, López-Olmedo / ORC ID: 0000-0002-2351-3699, Open ID: 102635503757035525450, CVU CONACYT ID: 1006679

DOI: $10.35429 /$ JCS.2019.7.3.5.12

Recibido: 12 de Enero, 2019; Aceptado 30 de Marzo, 2019

\section{Resumen}

En el presente trabajo se desarrolla una estación de maquinado CNC (Control Numérico Computarizado) para su posterior integración en un prototipo de celda de manufactura, considerado como un sistema mecatrónico. Para esto, se asume la tarea de realizar el diseño mecánico y de control de un centro de maquinado vertical CNC, con el único objetivo de mecanizar piezas con características específicas a partir del método de remoción de material. El desarrollo y diseño de la estación de maquinado CNC inició con la fabricación de la estructura mecánica a partir de materiales rígidos y adecuados para su factible construcción; fue necesario la impresión 3D de piezas para la sujeción de los motores a pasos y su respectivo control se logró al emplear la plataforma de software y hardware Arduino y Universal Gcode Sender, en conjunto con el módulo de control de motores a pasos. Posteriormente se realizan los ajustes necesarios y de programación para llevar a cabo las pruebas correspondientes de funcionamiento para el aseguramiento de un adecuado mecanizado de las piezas requeridas, mismo que marque la continuidad del proceso de integración de esta estación a un prototipo de celda de manufactura.

Estación de maquinado, CNC, Universal Gcode Sender.

\begin{abstract}
In this paper a $\mathrm{CNC}$ machining station (Computerized Numerical Control) is developed for its later integration into a manufacturing cell prototype, considered as a mechatronic system. For this, it is assumed the task of carrying out the mechanical and control design of a CNC vertical machining center, with the sole objective of machining parts with specific characteristics from material removal method. The development and design of a CNC machining station began with the manufacture of mechanical structure from rigid and suitable materials for its feasible construction; 3D printing of parts was necessary for fastening stepper motors used and its respective control was achieved by using Arduino software and hardware platform and Universal Gcode Sender, in conjunction with a stepper motor control module. Subsequently, necessary adjustments and programming are made to carry out corresponding functional tests and to ensure adequate machining of required parts, which marks continuity of integration process of this station to a manufacturing cell prototype.
\end{abstract}

Machining station, CNC, Universal Gcode Sender

Citación: JARA-RUIZ, Ricardo, RODRÍGUEZ-FRANCO, Martín Eduardo, LÓPEZ-ÁLVAREZ, Yadira Fabiola y LÓPEZOLMEDO, Leonardo Joaquín. Desarrollo de prototipo de estación de maquinado CNC. Revista de Simulación Computacional. 2019. 3-7: 5-12

\footnotetext{
* Correspondencia al Autor: (ricardo.jara@utna.edu.mx)

$\dagger$ Investigador contribuyendo como primer autor.
} 


\section{Introducción}

La introducción del control numérico computarizado (CNC) ha ampliado exponencialmente las aplicaciones de las maquinas industriales mediante la automatización programable, dichas maquinas logran hacer movimientos imposibles de realizar manualmente. El uso de la tecnología CNC ayuda a mejorar la calidad y producción de piezas en grandes cantidades y de gran dificultad, en este caso se genera el diseño de una máquina vertical $\mathrm{CNC}$ con el objetivo de la fabricación de piezas a partir de determinadas características. En el presente trabajo se describen las diferentes etapas del proceso para el desarrollo de un prototipo de estación de maquinado vertical $\mathrm{CNC}$ con dimensiones a menor escala que una unidad industrial, contemplando la parte mecánica, electrónica y de control, así como la configuración del hardware y software implementados. Al finalizar se muestras los resultados de funcionamiento obtenidos y las conclusiones a las que se llegó con el desarrollo del mismo.

\section{Manufactura}

La manufactura, en su sentido más amplio, es el proceso de convertir materia prima en productos. Incluye (1) el diseño del producto, (2) la selección de la materia prima y (3) la secuencia de procesos a través de los cuales será manufacturado el producto. La palabra manufactura se deriva del latín manufactus, que significa hecho a mano. La palabra manufactura apareció por primera vez en 1567 , y la palabra manufacturar en 1683. En el sentido moderno, la manufactura involucra la fabricación de productos a partir de materias primas mediante varios procesos, maquinarias y operaciones, a través de un plan bien organizado para cada actividad requerida.

\section{Manufactura integrada por computadora (CIM)}

Pocos desarrollos en la historia de la manufactura han tenidos un impacto más significativo que las computadoras, las cuales se utilizan ahora en una amplia gama de aplicaciones, incluyendo el control y la optimización de los procesos de manufactura, manejo de materiales, ensamble, inspección y prueba automatizada de los productos, control de inventarios, y numerosas actividades administrativas.
Empezando con los gráficos por computadora y el diseño y manufactura asistida por computadora, el uso de las computadoras se ha extendido a la manufactura integrada por computadora (CIM).

A continuación aparece un esquema de las aplicaciones principales de las computadoras en la manufactura:

a.

Control numérico por computadora

(CNC). Este es un método para controlar los movimientos de los componentes de las máquinas, mediante la inserción directa de instrucciones codificadas en forma de datos numéricos. El control numérico fue implementado por primera vez a principios de las década de 1950 siendo un adelanto de importancia en la automatización de máquinas.

b. Los sistemas de ensamble automatizados y robóticos están remplazando el costoso ensamble mediante operadores humanos. Los productos se diseñan o se rediseñan de manera que puedan ser ensamblados más fácilmente a máquina.

c. Sistemas de manufactura flexible (FMS). Esta metodología integra las celdas de manufactura en una unidad grande, toda ella con una interfaz con una computadora central. Los sistemas de manufactura flexible tienen el nivel más elevado de eficiencia, de complejidad y de productividad entre los sistemas de manufactura. Aunque son muy costosas, son capaces de producir partes con eficiencia en pequeños lotes y de modificar secuencias de manufactura de diferentes piezas de manera rápida; esta flexibilidad les permite hacer frente a cambios rápidos en la demande del mercado para diversos tipos de productos (Schmid, 2002).

\section{Configuración de robot cartesiano.}

Contiene tres grados de libertad y sus articulaciones son prismáticas en los tres ejes. De forma abreviada la configuración cartesiana se puede denominar como PPP, por el tipo de articulación. 
Las coordenadas asociadas son las cartesianas tal que cualquier punto en el espacio se describe mediante un vector de posición como $P_{i}=\left[x_{i}, y_{i}, z_{i}\right]^{T}$ como se muestra en la Figura 1 (Martín Hernández Ordoñez, 2015).

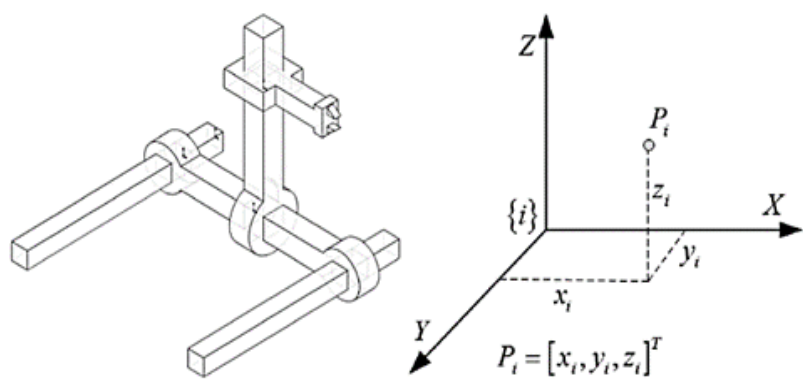

Figura 1 Configuración cartesiana

\section{Corte de material por medio de robots cartesianos}

El corte de materiales mediante robot es una aplicación reciente que cuenta con notable interés. La capacidad de reprogramación del robot y su integración en un sistema CIM (Manufactura Integrada por Computadora), hacen que aquel sea el elemento ideal para transportar la herramienta de corte sobre la pieza, realizando con precisión un programa de corte definido previamente desde un sistema de Diseño Asistido por Computadora (CAD)

(Antonio Barrientos, 2007).

\section{Programación de piezas asistida por computadora}

La programación de piezas asistida por computadora implica usar lenguajes de programación simbólicos, que determinan las coordenadas de puntos como esquinas, bordes y superficies de la pieza. Un lenguaje de programación es un método para comunicarse con la computadora; implica usar caracteres simbólicos. El programador describe, en este lenguaje, el componente que se va a procesar, y la computadora convierte esa descripción en comandos para la máquina de control numérico. Hoy, las piezas complicadas se maquinan con programas de maquinado basado en gráficas y asistido por computadora. Se crea una trayectoria de herramienta en un ambiente principalmente gráfico, parecido a un programa CAD. El programa o código de la máquina (Código G) se crea en forma automática (Serope Kalpakjian, 2002).

\section{Ácido poliláctico (PLA)}

Los avances recientes en la ciencia y la tecnología de materiales permiten contar con una variedad de métodos de síntesis de polímeros biodegradables. En el año de 1997, Cargill, empresa dedicada a negocios en el ámbito agropecuario, y Dow Chemical se asociaron para desarrollar un poliéster biodegradable, que se sintetizara a partir de dextrosa (glucosa) obtenida del maíz. Básicamente, estas empresas lograron transformar el azúcar en plástico denominado ácido poliláctico (PLA), comercializado bajo el nombre de NatureWorks. El PLA es el primer polímero sintético biodegradable obtenido a partir de recursos naturales renovables, con la enorme ventaja de presentar características similares al PET, ampliamente utilizado en envases de gaseosas y fibras textiles (Nudelman, 2004).

\section{Desarrollo}

\section{Etapa mecánica}

El diseño de la estructura mecánica del prototipo se define a partir de la configuración cartesiana, la cual constituye tres articulaciones prismáticas (PPP) y en efecto se establecen los ejes correspondientes $\mathrm{X}, \mathrm{Y}$ y Z.

El desplazamiento lineal de cada uno de los ejes se realiza por medio de tornillos sin fin y guías metálicas ubicadas a los costados de la plataforma empleando placas de acero y aluminio como soporte estructural (Figura 1).

Además, se realiza a través de impresión 3D utilizando material tipo PLA los soportes (Figura 2) donde se fijan los motores a pasos y las juntas (Figura 3) para el acople con los tornillos sin fin en cada uno de los ejes. Para el ensamble del soporte con la estructura mecánica son necesarios los componentes que forman parte de la base (Figura 4) que mantienen la linealidad y unión del motor con el elemento del eje. 


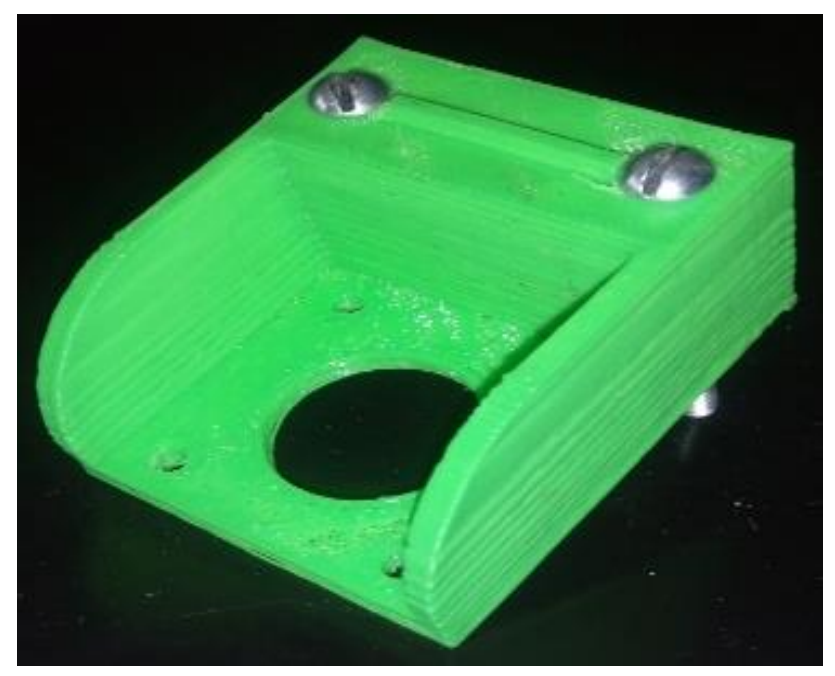

Figura 2 Soporte de motor a pasos

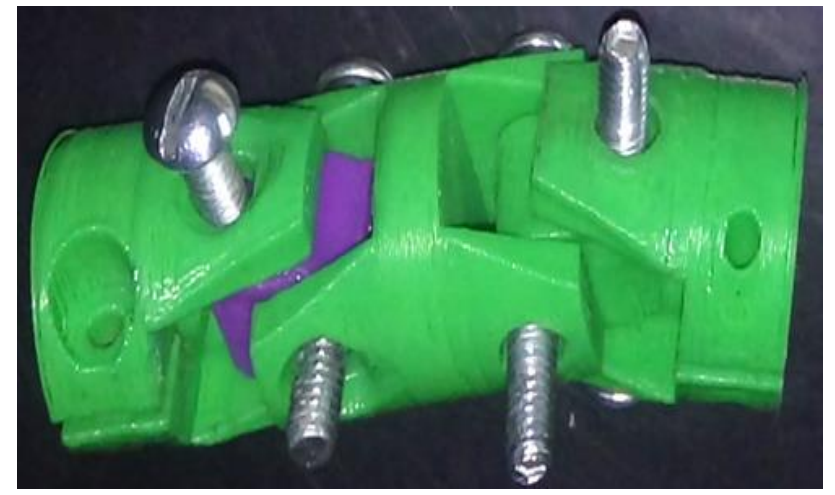

Figura 3 Junta

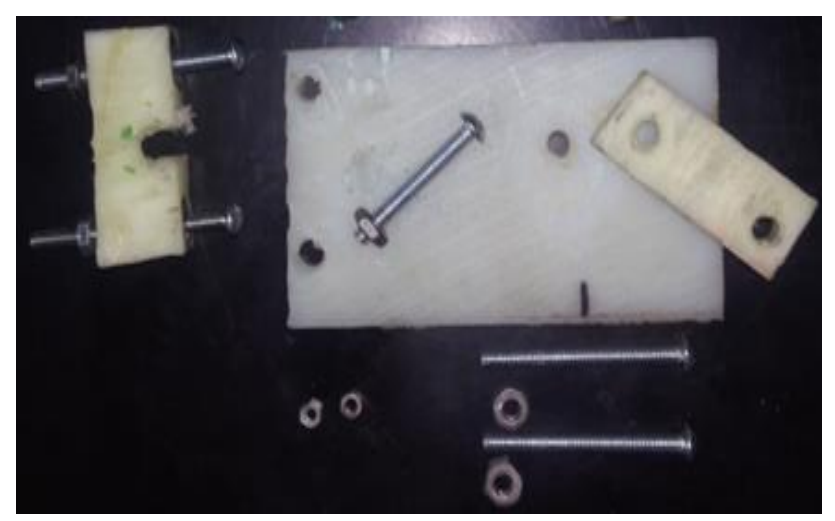

Figura 4 Componentes para la base

Teniendo los componentes necesarios se realiza el ensamble del motor a pasos con la base (Figura 5) que permite la transmisión del movimiento al elemento del eje correspondiente.

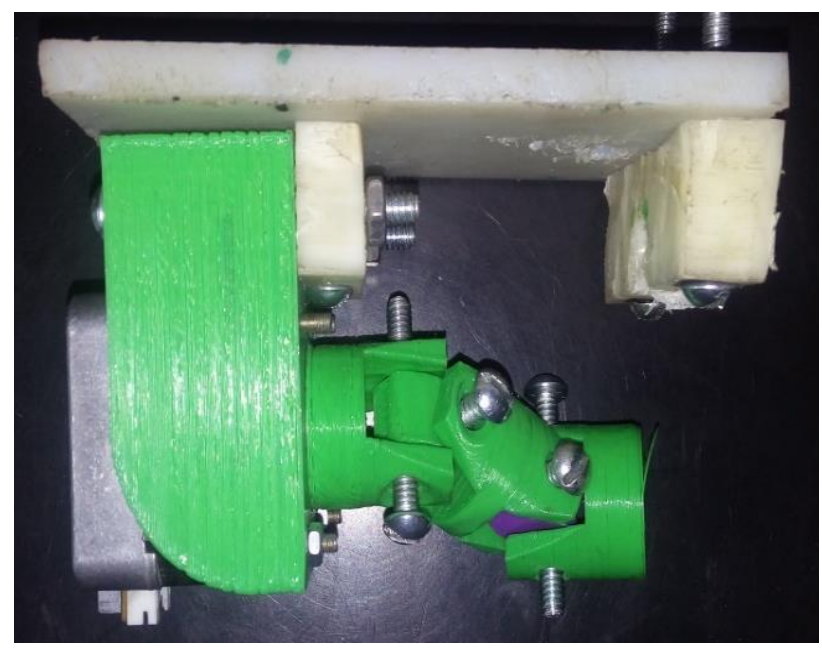

Figura 5 Ensamble de la base de transmisión

Posteriormente se procede a ensamblar la base de transmisión de cada eje (Figura 6, Figura 7 y Figura 8) y los tronillos sin fin acoplados a través de rodamientos a la estructura mecánica, los cuales cuenta con un sensor de final de carrera (limit switch) para delimitar el alcance mecánico.

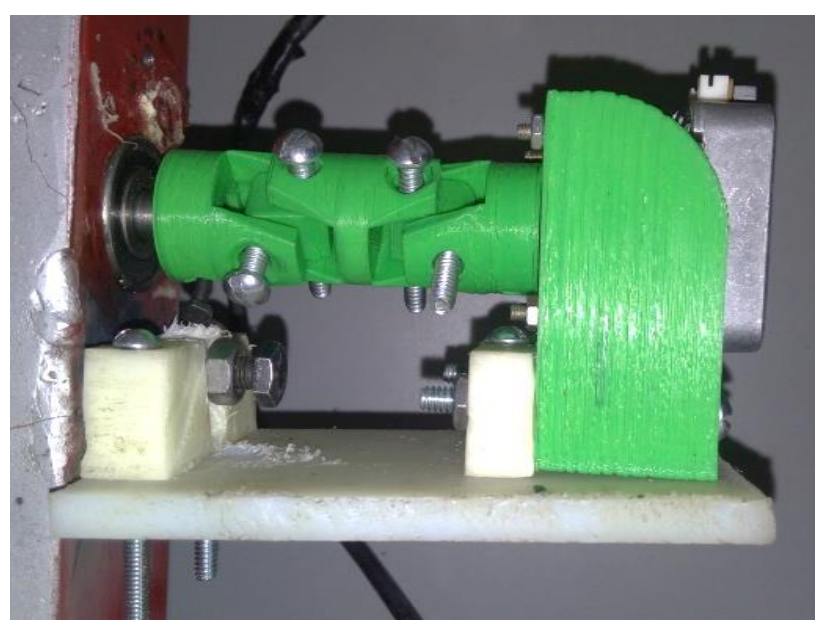

Figura 6 Eje $X$

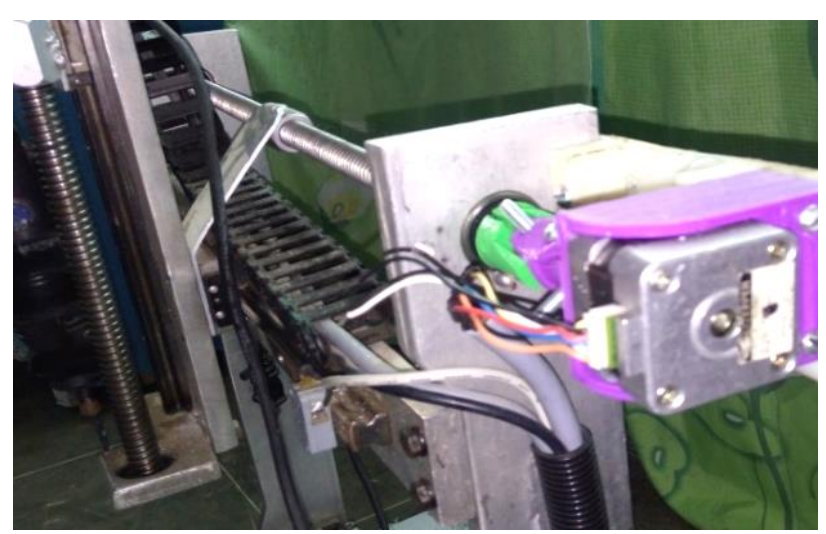

Figura 7 Eje Y 


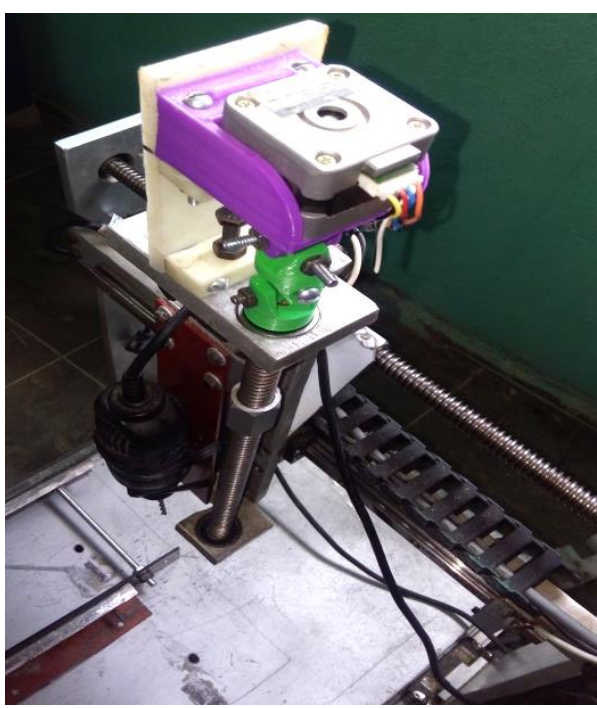

Figura 8 Eje Z

Finalmente se anexan a esta etapa la herramienta de trabajo tipo mototool como efector final (Figura 8) y el sistema de prensado de materia prima (Figura 9) que permite mantener en posición la pieza de material a maquinar. Para esto se emplea un motor de corriente directa unido a un tornillo sin fin, y este a su vez a un panel de acrílico que prensa el material hacía el panel fijo, el cual permite el ajuste manual para centrar la pieza a través de la plataforma.

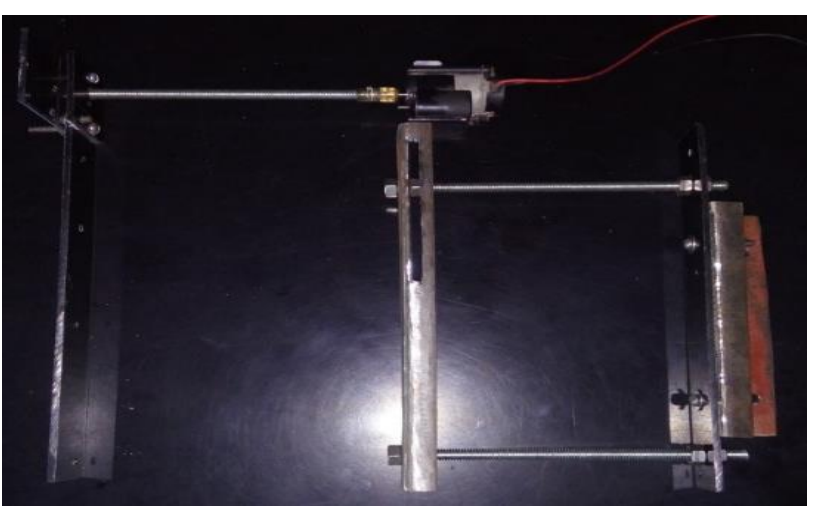

Figura 9 Sistema de prensado de material

\section{Etapa del sistema electrónico.}

Para esta etapa se emplean motores a pasos NEMA 17 tipo unipolares con fuerza radial de $1.8 \mathrm{~kg}$ y 200 pasos por vuelta $\left(360^{\circ}\right)$, es decir, $1.8^{\circ}$ por paso; gracias a estas características de los motores se consideran los apropiados para aplicaciones de esta índole. Para la conexión de los motores a pasos se contempla mantener la sincronía de pasos para lograr que los pulsos enviados sean los correctos para su óptimo funcionamiento, a lo cual la identificación de las bobinas por parte de los motores se muestra en la Tabla 1.

\begin{tabular}{|l|l|l|l|}
\hline Bobina & \multicolumn{1}{|c|}{ Eje X } & \multicolumn{1}{c|}{ Eje Y } & \multicolumn{1}{c|}{ Eje Z } \\
\hline A & Amarillo & Rojo & Amarillo \\
\hline B & Azul & Amarillo & Azul \\
\hline C & Rojo & Azul & Rojo \\
\hline D & Naranja & Verde & Naranja \\
\hline
\end{tabular}

Tabla 1 Identificación de bobinas de cada motor a pasos acorde al color de cable

Cabe mencionar que no es necesario conectar los cables de tierra $(+\mathrm{Vcc})$, esto por especificaciones técnicas del módulo CNC.

Seguido se calcula la cantidad de pasos necesarios para poder desplazarse un milímetro en cada eje.

$P M=\frac{p t e}{p d * 10}$

Donde:

$P M$ : pasos por milímetro.

pte: pasos totales por eje.

pd : posible desplazamiento.

Al aplicar la ecuación (1) se obtienen los resultados de los pasos por milímetro correspondientes a cada eje (Tabla 2).

\begin{tabular}{|l|c|l|}
\hline Eje & Cálculo & Pasos por mm \\
\hline $\mathrm{X}$ & $P M=\frac{10810}{23 * 10}$ & 47 \\
\hline $\mathrm{Y}$ & $P M=\frac{13670}{29.2 * 10}$ & 47 \\
\hline $\mathrm{Z}$ & $P M=\frac{8420}{18 * 10}$ & 47 \\
\hline
\end{tabular}

Tabla 2 Pasos por milímetro de cada eje

Respecto al módulo $\mathrm{CNC}$, es necesario realizar un ajuste a los controladores (drivers) pololu A4988 para limitar la corriente máxima soportada por los motores a pasos mediante el potenciómetro que se muestra en la Figura 10.

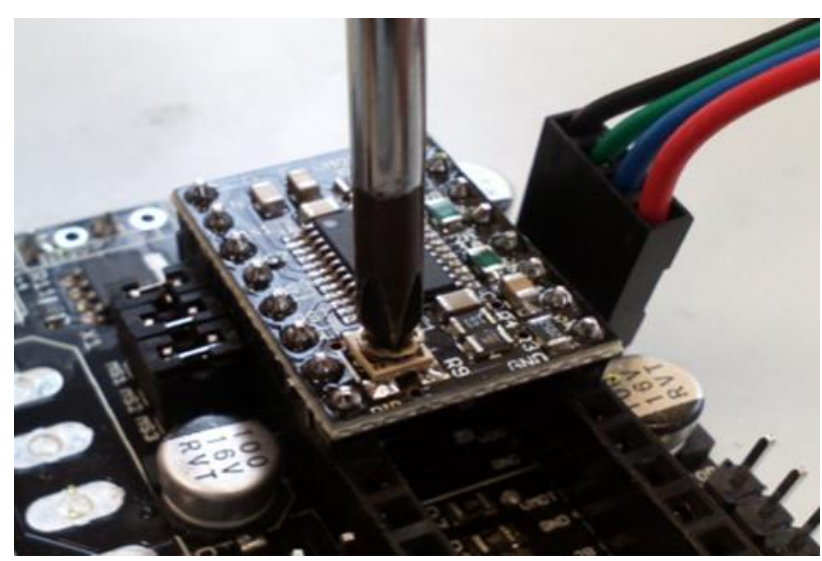

Figura 10 Ajuste de corriente en pololu A4988

JARA-RUIZ, Ricardo, RODRÍGUEZ-FRANCO, Martín Eduardo, LÓPEZ-ÁLVAREZ, Yadira Fabiola y LÓPEZ-OLMEDO, Leonardo Joaquín. Desarrollo de prototipo de estación de maquinado CNC. Revista de Simulación Computacional. 2019 
La forma de calibrar los pololu a una corriente apta para cada motor se hace por medio de la aplicación de una formula la cual es:

$$
I_{\text {TripMAX }}=V_{R E F} /(8 * R s)
$$

Donde:

$I_{\text {TripMAX }}$ es la corriente máxima suministrada en amperes (A).

$R s$ es la resistencia de detección en ohms $(\Omega)$.

$V_{R E F}$ es el voltaje de referencia $(\mathrm{V})$.

Considerando una corriente máxima de 1.4 A y una resistencia de detección de $0.1 \Omega$ al aplicar la ecuación (2) para determinar $V_{R E F}$ se tiene:

$$
\begin{aligned}
& V_{R E F}=1.4 A *(8 * 0.1 \Omega) \\
& V_{R E F}=1.12 \mathrm{~V}
\end{aligned}
$$

Por especificaciones técnicas para la configuración de paso completo en los motores se considera alcanzar el $70 \%$ de la corriente a suministrar por el controlador.

$$
\begin{aligned}
& V_{R E F}=1.12 * .7 \\
& V_{R E F}=0.78 \mathrm{~V}
\end{aligned}
$$

Se verifica con el instrumento de medición (multímetro) que el voltaje de referencia de cada controlador sea el indicado de acuerdo al valor determinado por los cálculos.

Posterior al ajuste de los controladores instala el módulo CNC y la placa Arduino Uno en el gabinete de control (Figura 11).

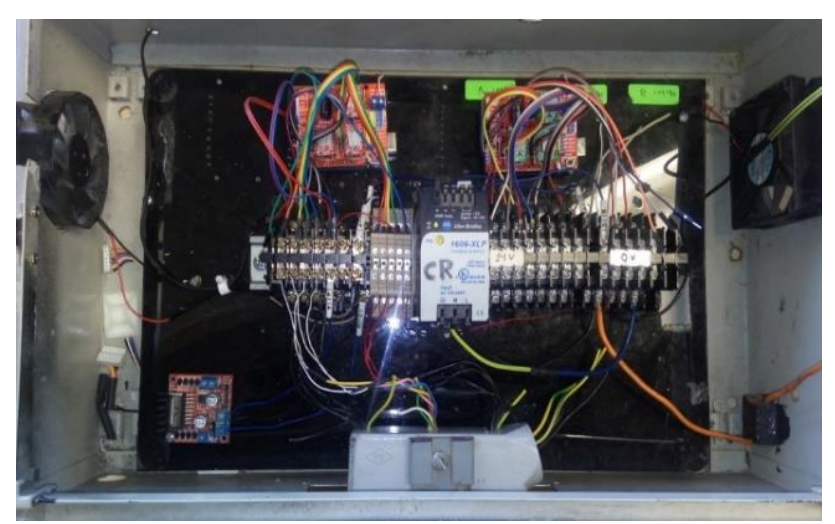

Figura 11 Gabinete de control

\section{Etapa de Control}

La etapa de control se desarrolla a partir del software Universal Gcode Sender el cual se conecta por el puerto serial hacia el hardware donde previamente se cargó el firmware GRBL el cual realiza la interpretación del código $\mathrm{G}$ a comandos para enviar las secuencias a los motores a pasos a través del módulo CNC.

Para verificar si la secuencia y relación de pasos por milímetros es la correcta se procede a realizar pruebas, es decir, se enviar 100 milímetros de manera manual empleando el software Universal Gcode Sender a los ejes tanto positivamente como negativamente para después se verifica con un patrón de medición si el desplazamiento es el establecido.

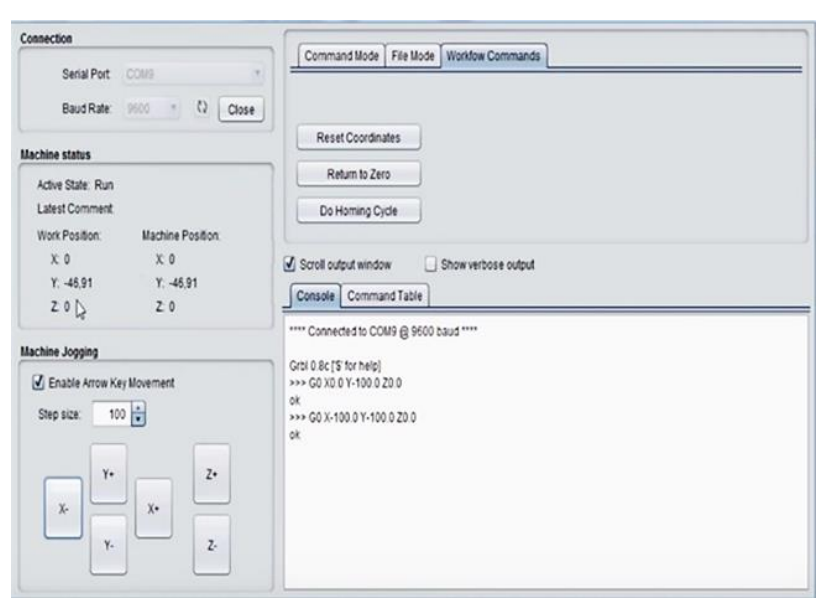

Figura 12 Pruebas de desplazamiento manuales con Universal Gcode Sender

En la Figura 12 se puede observar en el apartado Step size el envío de datos correspondiente a los $100 \mathrm{~mm}$ de desplazamiento indicados, en el apartado de Console se visualiza el registro de los mismos y en el apartado de machine status se muestra el avance en los ejes pertinentes.

Ya confirmado el funcionamiento del centro de maquinado vertical $\mathrm{CNC}$, se procede a cargar el archivo que contiene el código G generado correspondiente al diseño de la pieza a maquinar (Figura 13) empleando el software Universal Gcode Sender (Figura 14). 


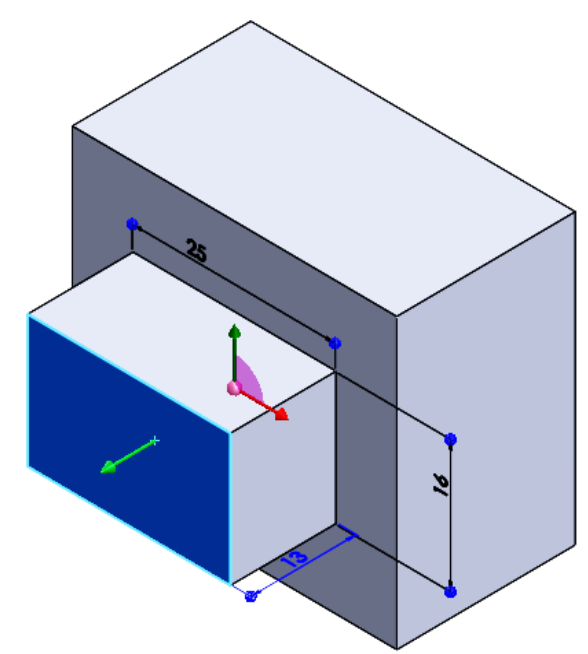

Figura 13 Pieza diseñada en Solidworks

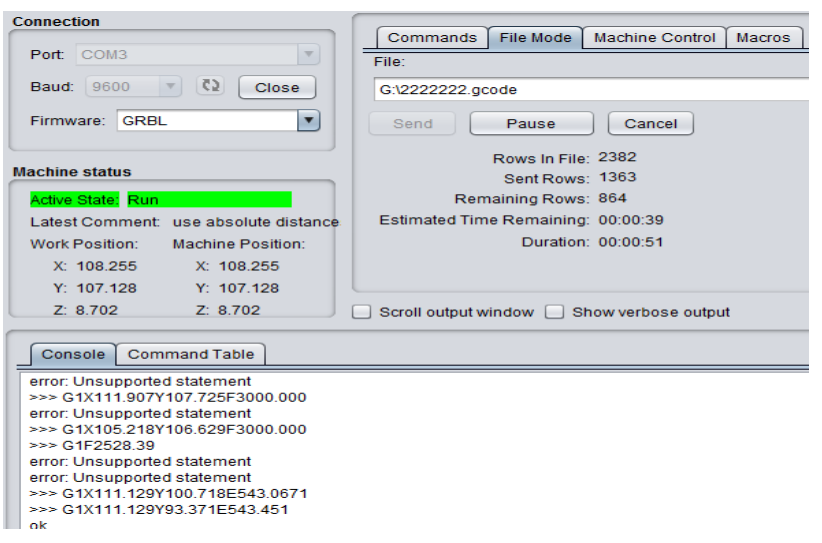

Figura 14 Envío del código $G$ a través del software Universal Gcode Sender

Enseguida se observa a través de la herramienta G-Code Visualizer el estado de avance correspondiente al código G (Figura 15) y el proceso de maquinado por remoción de material (Figura 16) de la pieza.

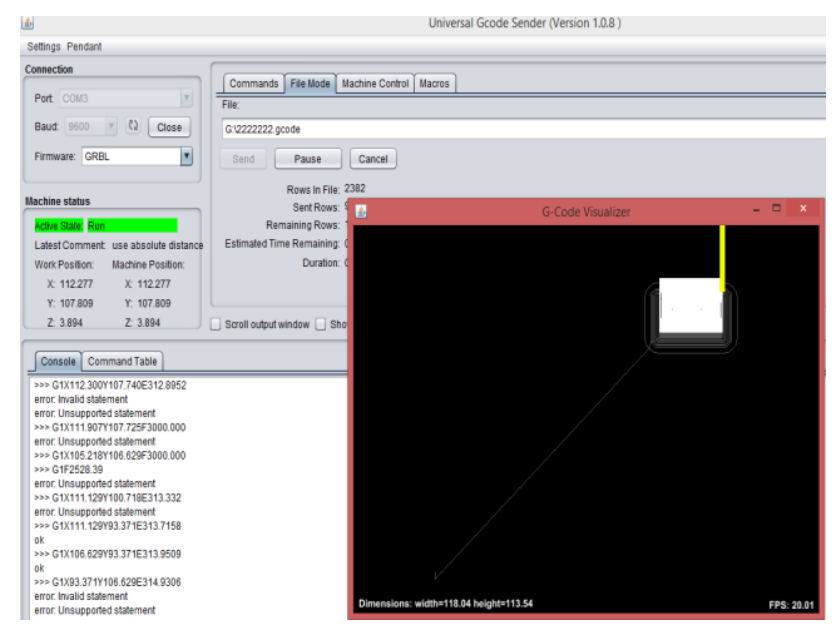

Figura 15 Herramienta G-Code Visualizer

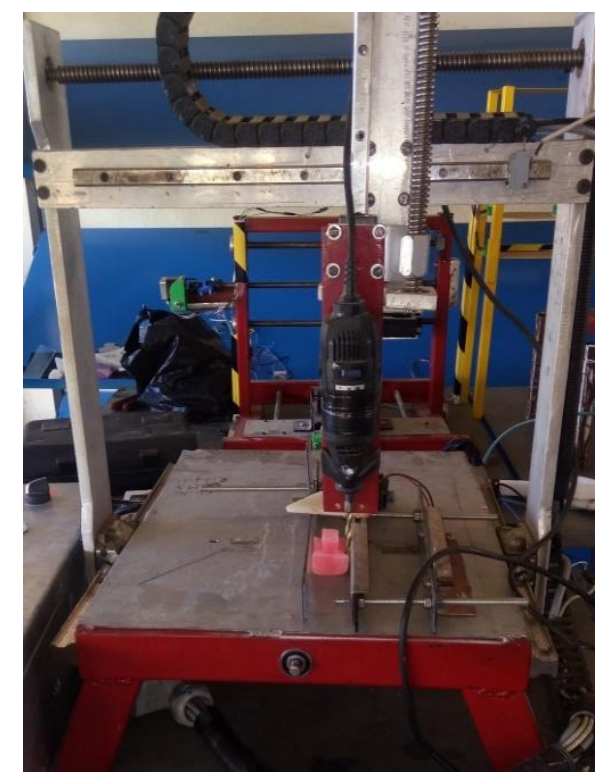

Figura 16 Proceso de maquinado por remoción de material

\section{Resultados}

Los resultados obtenidos durante el proceso de desarrollo de las etapas contempladas para el presente trabajo fueron favorables, ya que se logró un diseño estructural adecuado al conseguir un desplazamiento continuo al trasmitir el movimiento hacía los ejes coordenados, así como una correcta comunicación hardware/software para el control del prototipo.

Concluidas las etapas de fabricación y pruebas de funcionamiento, se obtiene como primer resultado el ensamble completo de la estación de maquinado vertical (Figura 17 y Figura 18), la cual cuenta con dimensiones estructurales de $630 \mathrm{~mm}$ de ancho, $640 \mathrm{~mm}$ largo y $900 \mathrm{~mm}$ de alto; y con una capacidad de área de trabajo de:

- $\quad$ Carrera máxima en eje X: $230 \mathrm{~mm}$.

- $\quad$ Carrera máxima en eje Y: $245 \mathrm{~mm}$.

- $\quad$ Carrera máxima en eje Z: $180 \mathrm{~mm}$. 


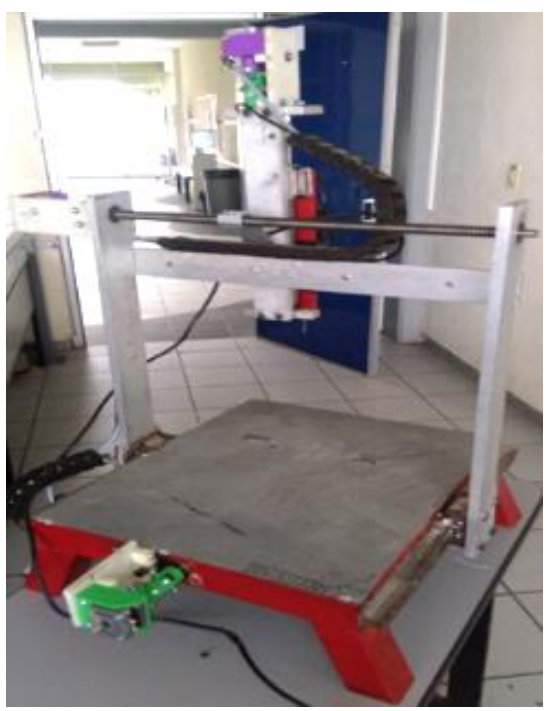

Figura 17 Estación de maquinado vertical

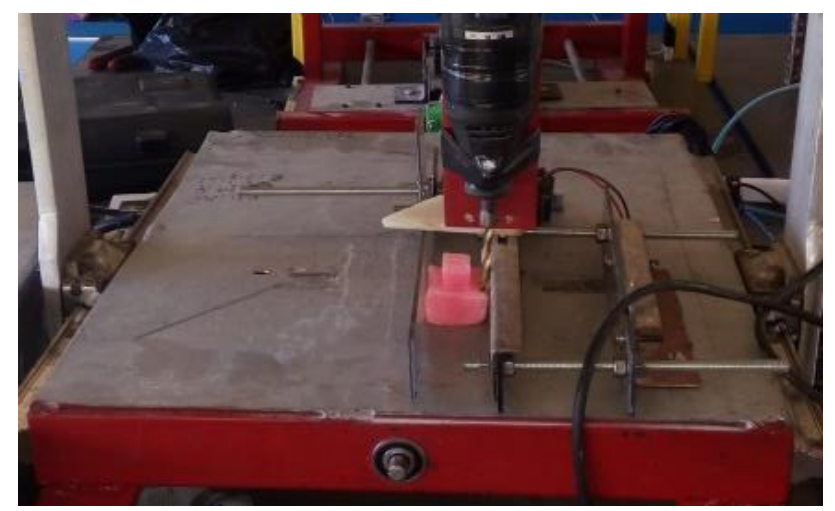

Figura 18 Plataforma de la estación de maquinado vertical

Finalmente por medio del método de remoción de material se logra el maquinado de piezas, siendo que la Figura 19 muestra el resultado obtenido a partir del diseño en Solidworks (Figura 13) empleando el prototipo de estación de maquinado vertical CNC.

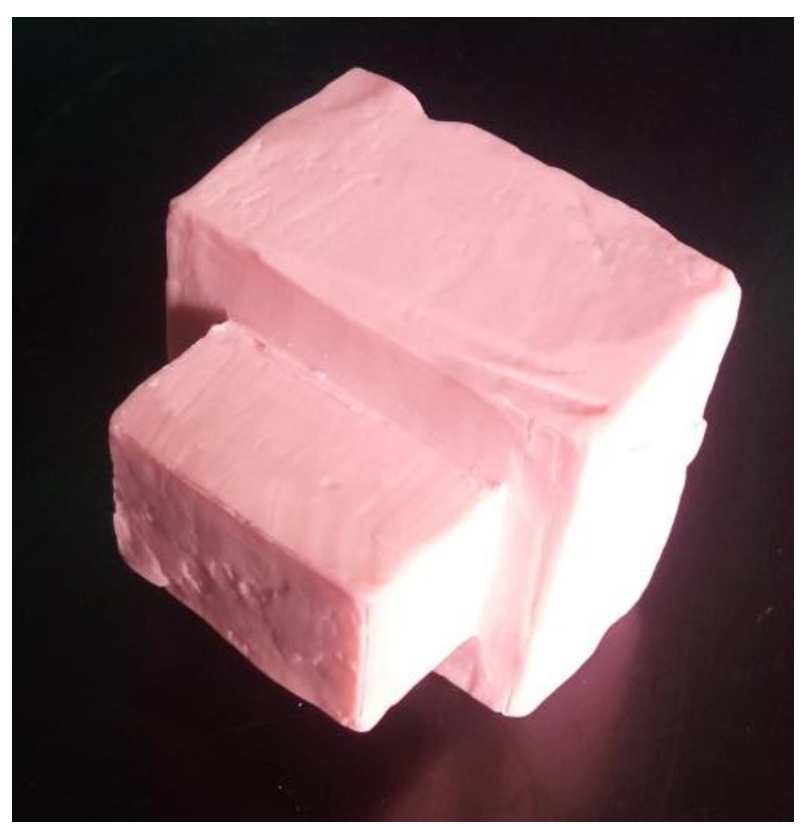

Figura 19 Pieza maquinada por remoción de material
Alcanzados estos resultados se contempla como trabajo a futuro dar la continuidad al proceso que permita la integración de esta estación de trabajo a un prototipo de celda de manufactura.

\section{Conclusiones}

El desarrollo del presente trabajo permitió llevar a cabo una sinergia entre disciplinas de diferentes campos de conocimiento resultando de gran relevancia e importancia en el fortalecimiento de las áreas de aplicación y desarrollo de sistemas mecatrónicos.

Cumplir con las etapas correspondientes al prototipo y las metas planteadas por el equipo de trabajo llevó a conocer y seleccionar las tecnologías más adecuadas e indicadas acorde a las necesidades de la aplicación; el conseguir integrar los diferentes elementos mecánicos, electrónicos y de control para la fabricación de la estructura mecánica y su correcto funcionamiento respectivamente al convertir instrucciones o comandos a desplazamientos en los ejes transformándose por medio de la herramienta de trabajo en remoción de material para obtener como resultado la pieza requerida. A lo cual el esfuerzo implicado se traduce en resultados óptimos en implementación y funcionamiento del prototipo de estación de maquinado vertical. Además, el involucramiento de estudiantes de nivel superior aporta en gran medida al enriquecimiento y adquisición de competencias necesarios para su desarrollo profesional.

\section{Referencias}

Antonio Barrientos, L. F. (2007). Fundamentos de robótica. McGraw-Hill.

Martín Hernández Ordoñez, M. B. (2015). Robótica: Análisis, modelado, control e implementación. México: OmniaScience.

Nudelman, N. (2004). Química sustentable. Argentina: EdicionesUNL.

Schmid, S. K. (2002). Manufactura, ingeniería y tecnología. México: Pearson Educación.

Serope Kalpakjian, S. R. (2002). Manufactura, ingeniería y tecnología. México: Pearson Educación. 